Article

\title{
Exploring the Distribution of Traffic Flow for Shared Human and Autonomous Vehicle Roads
}

\author{
Huanping $\mathrm{Li}^{1}{ }^{1}$, Jian Wang ${ }^{1}$, Guopeng Bai ${ }^{2}$ and Xiaowei Hu ${ }^{1}$ \\ 1 School of Transportation Science and Engineering, Harbin Institute of Technology, Harbin 150090, China; \\ wang_jian@hit.edu.cn (J.W.); xiaowei_hu@hit.edu.cn (X.H.) \\ 2 Department of Civil and Environmental Engineering, University of Macau, Taipa, Macau 999078, China; \\ yc07450@umac.mo \\ * Correspondence: 15202217597@163.com or 15B932007@hit.edu.cn
}

Citation: Li, H.; Wang, J.; Bai, G.; Hu, $X$. Exploring the Distribution of Traffic Flow for Shared Human and Autonomous Vehicle Roads. Energies 2021, 14, 3425. https://doi.org/ $10.3390 /$ en14123425

Academic Editor: Calin Iclodean

Received: 2 May 2021

Accepted: 7 June 2021

Published: 10 June 2021

Publisher's Note: MDPI stays neutral with regard to jurisdictional claims in published maps and institutional affiliations.

Copyright: (c) 2021 by the authors. Licensee MDPI, Basel, Switzerland. This article is an open access article distributed under the terms and conditions of the Creative Commons Attribution (CC BY) license (https:// creativecommons.org/licenses/by/ $4.0 /)$.

\begin{abstract}
In order to explore the changes that autonomous vehicles would bring to the current traffic system, we analyze the car-following behavior of different traffic scenarios based on an anti-collision theory and establish a traffic flow model with an arbitrary proportion $(p)$ of autonomous vehicles. Using calculus and difference methods, a speed transformation model is established which could make the autonomous/human-driven vehicles maintain synchronized speed changes. Based on multi-hydrodynamic theory, a mixed traffic flow model capable of numerical calculation is established to predict the changes in traffic flow under different proportions of autonomous vehicles, then obtain the redistribution characteristics of traffic flow. Results show that the reaction time of autonomous vehicles has a decisive influence on traffic capacity; the $q-k$ curve for mixed human/autonomous traffic remains in the region between the $q-k$ curves for $100 \%$ human and $100 \%$ autonomous traffic; the participation of autonomous vehicles won't bring essential changes to road traffic parameters; the speed-following transformation model minimizes the safety distance and provides a reference for the bottom program design of autonomous vehicles. In general, the research could not only optimize the stability of transportation system operation but also save road resources.
\end{abstract}

Keywords: autonomous vehicles; arbitrary proportion; speed change; redistribution

\section{Introduction}

Increasing the proportion of autonomous vehicles will lead to a change of travel mode from human vehicles to autonomous vehicles, which will bring significant variation to traditional traffic and have a great influence on traffic flow characteristics, thus affecting the overall optimization design of the road network. As the technology matures, some cities are starting to test autonomous vehicles on the road. With autonomous vehicles gradually being accepted by the public, the precision and communication capabilities between autonomous vehicles and cloud-computers will allow new traffic behaviors to enhance the capacity of the road network. Moreover, the emergence of autonomous vehicles could realize some idealized assumptions of previous traffic models, for example, autonomous vehicles could keep a constant speed and deal with traffic behavior completely rationally.

The smaller reaction time of autonomous vehicles could reduce the car-following distance between vehicles and increase road capacity [1]. Compared with human-driven vehicles, they are less susceptible to adverse road conditions. The research finds that the participation of autonomous vehicles on human-driven roads could greatly improve travel efficiency when the road is not congested. However, on shared roads, it is still difficult if vehicles can not be guided in time to avoid traffic congestion. If the research systems are all autonomous vehicles, the travel efficiency could be increased to more than twice of all human-driven vehicles under the same conditions, as shown in as shown in experimental analysis part. If the running time of the mixed system is long enough, the autonomous vehicles could adjust to the optimal state without the interference of human-driven vehicles 
and the travel efficiency of the system could still increase as the proportion of autonomous vehicles increases. This is one of the reasons why it takes a while for autonomous vehicles to be fully accepted by travelers.

British scholars Lighthill and Whitham [2] proposed the hydrodynamics theory in 1955 which studied the changes of traffic flow under the condition of high traffic density and proposed the hydrodynamics simulation theory. Subsequently, Richards [3] independently proposed a similar traffic flow theory. Later scholars called this continuous medium describing the traffic flow as an LWR theoretical model. After the theory was widely used, G.C.K. Wong and S.C Wong [4] promoted a model to improve the accuracy of their description of realistic traffic and established a dynamic model of a variety of vehicle models. Based on multi-hydrodynamic theory, the paper establishes a mixed traffic flow model to predict the changes in traffic flow under different proportions of autonomous vehicles, then obtains the redistribution characteristics of traffic flow.

There are several different types of car-following models [5,6]. For example, some models can predict how the driver would make changes in acceleration according to the vehicle in front. Newell [7] simplified the car-following model in earlier years and the simplified model is consistent with the fluid dynamics theory but it does not include reaction time. According to the anti-collision theory of Kometani and Sasaki [8], a car-following model with reaction time is established to predict the allowable space at a given speed. The decisive factor in predicting headway with speed is density. The results have shown that the model is consistent with the triangle theory of Newell and Yperman et al. $[9,10]$. Based on this we used the calculus and difference methods to establish a speed transformation model which could make autonomous and human-driven vehicles maintain synchronized speed changes.

Current research on autonomous vehicles mostly focuses on intersections, safety, comfort experiences [11], car-following behavior between vehicles or shared travel and autonomous/human-driven vehicles driving in separate lanes or lane-changing under mixed traffic [12-14]. The role of autonomous vehicles in a smart city, intelligent transportation and shared travel is also one of the main research directions, at present. Some scholars have studied the impact of autonomous vehicles on society in the long, short and mediumterm. If the current system is all autonomous vehicles, it can bring huge potential benefits to society such as reducing air pollution and improving work efficiency. In the short term, especially with the COVID-19 outbreak in 2019 and 2020, autonomous vehicles could not only reduce the risk of transmission and avoid unnecessary travel contacts but also could spontaneously pick the medical staff up. In the medium term, it can affect economic development: the use of autonomous vehicles could trigger new investment opportunities and new markets, and the government could make macro-economic adjustments and change corresponding with congestion strategies. In the long run, efficient, interconnected and automated travel by autonomous vehicles could reduce pollution, improve air quality and mitigate the impact of climate change on the environment. However, previous literature studies on autonomous vehicles are mostly micro experiments, there are few macroscopic experiments on the impact of autonomous vehicles gradually driving on the road. Based on this, the purpose of this paper is to develop a shared road traffic flow model for any scenario with different proportions of autonomous vehicles. The main contributions are as follows:

(1) Analyzed the possible changes in traffic parameters brought by autonomous vehicles;

(2) Using collision avoidance theory, the traffic flow models are analyzed respectively and makes assumptions based on the triangular diagram proposed by Newell;

(3) A car-following model is established in which the autonomous vehicle can stably follow human-driven vehicles in any scene;

(4) Establish a mixed traffic flow analysis model by relaxing the velocity variable;

(5) Based on the hydrodynamic theory, a mixed traffic flow model with an arbitrary proportion of autonomous vehicles is established. 
The rest of the article is organized as follows: the second part explains the research background of the article. The third part presents the consistency between the multihydrodynamic mechanics and the mixed traffic flow model, then makes assumptions about the model to be established. The fourth part is the main content of the paper, which establishes a traffic flow model of any scene with an arbitrary proportion of autonomous vehicles. The fifth section studies the change of traffic flow when the proportion of autonomous vehicles changes in a road segment and gives the calculation result analysis of an example. The sixth part summarizes the article, points out the deficiencies of the research and the prospects for the follow-up work.

\section{Research Backgrounds}

Based on the general project of NSFC, "Research on the transportation network design theory based on vehicle automatic driving cloud plan", we discussed changes in road traffic flow when autonomous vehicles gradually increase until they occupy a dominant position in traffic.

Marked by the release of Google's self-driving car program, research on self-driving cars has entered a new era. The autonomous vehicle, also known as the driverless vehicle, is used as an independent vehicle that could sense the surrounding environment and automatically navigate without manual operation to meet the needs of human transportation. As autonomous vehicles are equipped with forward collision warning systems, automatic braking technology and other, unmanned driving automation technologies, they have the ability to reduce the risk of random and subjective behavior during driving. Autonomous vehicles are capable of reducing traffic accidents, reducing traffic congestion and increasing road capacity So, autonomous vehicles are the future of transportation development.

In the process of the change from coexistence to the dominance of autonomous vehicles and human-driven vehicles, the existing traffic theories and traffic assignment methods may not be able to fully adapt to future traffic practice due to changes in the path selection behavior of autonomous vehicles. How to make the current transportation system follow the development of smart cities and smart transportation is an urgent problem to be solved. This paper conducts a more detailed study of autonomous vehicles on the impact of the traffic flow at the core of the transportation system. The research could provide the most necessary basic reference for the distribution of road network traffics and road network design after autonomous vehicles driving on the road. At the same time, it can provide the necessary reference and basis for issues such as operation mode selection, overall inventory and traffic diversion, which is of great significance for autonomous vehicles on the road [15].

\section{Modeling Theoretical Foundation}

This section mainly introduces the extension of the hydrodynamic theory from a single type to a multiclass hydrodynamic theory, which means multiple vehicle types. The model is constructed according to the principle of calculus and backward Taylor expansion. The focus is on autonomous vehicles and human-driven vehicles in this paper.

\subsection{Multiple Hydrodynamic Theory}

Hydrodynamic mechanics is one of the main research methods of traffic flow theory. It uses the basic principle of hydrodynamic mechanics to establish the continuity equation of traffic flow by simulating the continuity equation of fluid. The theory abstracts the traffic flow wave by comparing the change of traffic flow density to the fluctuation of water waves. When the road traffic conditions and density change, the propagation of traffic waves are generated in the traffic flow. By analyzing the propagation speed of the traffic flow, the relationship between the flow, density and speed is found and the congestiondissipation process of the traffic flow is described. It is sometimes called hydrodynamics simulation theory. The multiple hydrodynamic theory in this paper is relative to the single hydrodynamics that focused on only one type of vehicle. Multiple hydrodynamic theory 
in this paper means there is more than one type of vehicle in the model and mainly refers to autonomous vehicles and human-driven vehicles.

Suppose set $M$ is the class of vehicles, $M \in\{1,2,3, \ldots \ldots, N\}, k_{m}(x, t)$ is the density of vehicle class $m$ at a space-time point $(x, t)$. Then the total density is:

$$
k(x, t)=\sum_{m \in M} k_{m}(x, t)
$$

Similarly, the class-specific flow $q_{m}$ at space-time point $(x, t)$ is:

$$
q_{m}=q_{m}(x, t)=u\left(\frac{k_{1}}{k}, \cdots, \frac{k_{M}}{k}\right) k_{m}(x, t)
$$

The function $u\left(\frac{k_{1}}{k}, \cdots, \frac{k_{M}}{k}\right)$ is the possible velocity of a vehicle with proportions of $\frac{k_{1}}{k}, \cdots, \frac{k_{M}}{k}$.

As with Daganzo [16-18], we show that these transition flows are consistent with the multiclass hydrodynamic theory. Let $k_{m} / k$ be the proportion of density occupied by specific traffic flow. Suppose that, under the same conditions, vehicles of the same class travel at the same speed and $k>0$ [1].

The speed is limited by free flow velocity, traffic volume and backward wave propagation:

$$
u\left(k_{1}, \cdots, k_{M}\right)=\min \left\{u_{f}, \frac{q^{\max }\left(\frac{k_{1}}{k}, \cdots, \frac{k_{M}}{k}\right)}{k}, w_{a}\left(\frac{k_{1}}{k}, \cdots, \frac{k_{M}}{k}\right)\left(\frac{k_{j a m}-k}{k}\right)\right\}
$$

where $u_{f}$ is the free-flow speed, $w_{a}\left(\frac{k_{1}}{k}, \ldots, \frac{k_{M}}{k}\right)$ is the backwards wave speed, $q^{\max }\left(\frac{k_{1}}{k}, \ldots, \frac{k_{M}}{k}\right)$ is the traffic flow when the proportions of each class in density are $\frac{k_{1}}{k}, \cdots, \frac{k_{M}}{k}$, and $k_{j a m}$ is the density of jam. According to $q=k u$, then:

$$
q_{m}(x, t)=\frac{k_{m}}{k} \min \left\{u_{f} k, q^{\max }\left(\frac{k_{1}}{k}, \cdots, \frac{k_{M}}{k}\right), w_{a}\left(\frac{k_{1}}{k}, \cdots, \frac{k_{M}}{k}\right)\left(k_{j a m}-k\right)\right\}
$$

Total traffic flow:

$$
q(x, t)=\sum_{m \in M} q_{m}(x, t)
$$

There are three traffic flow-limiting factors for class $m$ vehicles: (1) the speed of classspecific vehicles; (2) proportional share of class-specific; (3) proportional share of crowded flow. In this paper, it is assumed that autonomous vehicles and human-driven vehicles have the same physical characteristics such as vehicle length $l$ and maximum speed, so $k_{j a m}$ is independent of vehicle class.

In order to maintain the continuity of the research object, the flow conservation equation is satisfied for any $m \in M[5]: \partial q_{m}(x, t) / \partial x=-\partial k_{m}(x, t) / \partial t$.

\subsection{Model Assumptions}

In conventional hydrodynamic theory, the occupancy rate of a certain vehicle will change with time and space which may lead to the occurrence of none-first-come-firstserved phenomenon in the system. In order to avoid a certain class amount of traffic at the end of the road becoming a transitional flow with a higher proportion, the article assumes that the density is uniformly distributed. As stated by Blumberg and Bar-Gera [19], a single type of traffic flow may also violate the first-come, first-served rule. Since the total transition flow of discrete vehicles is continuous it could be regarded as a continuous flow. Therefore, the article has the following assumptions:

1. Autonomous vehicles and human-driven vehicles have the same physical characteristics, such as vehicle length $l$ and free-flow speed;

2. Vehicles of the same class travel at the same speed; 
3. The model is suitable for multiple types of vehicles. The focus of this article is autonomous vehicles and human-driven vehicles;

4. Different types of autonomous vehicles have different reaction times, so the vehicles will respond to different car-following behaviors;

5. The density of specific vehicles in the system is uniformly distributed.

\section{Modeling and Analysis}

In this section, based on kinematics theory and different vehicle reaction times, a car-following model that could predict the speed-density relationship is established. The model does not include other influencing factors, such as road traffic conditions, and can predict changes in traffic flow that are composed of different classes of vehicles. The influence of reaction time on capacity could be used to extend the traffic characteristics of road sections to the actual urban road network model. Although autonomous vehicles are less affected by changes in road traffic conditions than drivers, it is assumed that they are affected the same when establishing a shared road model with a different proportion of autonomous driving. Other factors affecting traffic volume are included in the model described in Part Three.

\subsection{Human Driving Traffic Flow Model}

The car-following behavior of human-driven vehicles in the traffic system has three main characteristics: (1) Restriction: the speed of the rear vehicle should not be greater than the speed of the preceding vehicle for a long time and there must be sufficient braking distance between vehicles; (2) Hysteresis: The rear vehicle needs reaction time to adapt to the speed change of the preceding vehicle; (3) Transitivity: Once the first vehicle changes its running state, its effect will be transmitted backward, one by one, until the last car in the fleet.

Since the acceleration of the linear following vehicle is only a function of the relative speed of the two vehicles, considering the complexity of the vehicle driven by a human, a nonlinear car-following model with sensitivity coefficient $\lambda$ is selected. According to Leutzbach's research [20]:

$$
\ddot{x}_{n-1}(t+\Delta t)=\lambda\left[\dot{x}_{n}(t)-\dot{x}_{n-1}(t)\right]
$$

$\Delta t$ is the reaction time, $\lambda$ is the sensitivity coefficient:

$$
\lambda=\lambda_{0} \frac{\dot{x}_{n+1}^{\alpha}(t+\Delta t)}{\left[x_{n}(t)-x_{n+1}(t+\Delta t)\right]^{\beta}}
$$

where $\alpha \geq 0, \beta \geq 0$. In a nonlinear $(\alpha, \beta)$ model, $\alpha$ and $\beta$ are constants for a certain model. In the model we use $(0,1.5)$ then the car-following model could be obtained [21]:

$$
\ddot{x}_{n+1}(t+\Delta t)=\lambda_{0} \frac{\left[\dot{x}_{n}(t)-\dot{x}_{n+1}(t)\right]}{\left[x_{n}(t)-x_{n+1}(t+\Delta t)\right]^{1.5}}
$$

Equivalent to:

$$
\dot{u}_{n+1}(t+\Delta t)=\lambda_{0} \frac{\dot{s}_{n+1}}{s_{n+1}^{1.5}}
$$

where $u_{n+1}$ means the velocity of the $(n+1)$ th vehicle. $s_{n+1}=x_{n}(t)-x_{n+1}(t)$ is the car-following distance between the $(n+1)$ th vehicle and the $n$th vehicle, as shown in the Figure 1 below: 


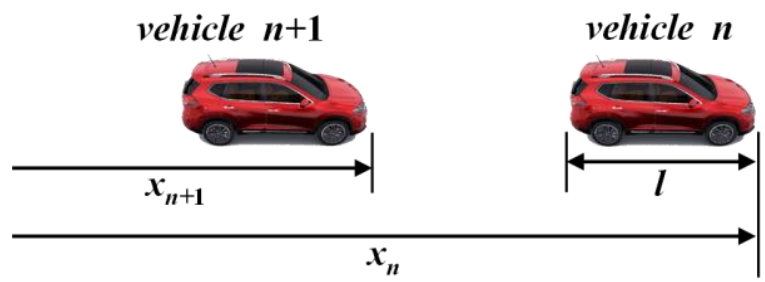

Figure 1. Schematic diagram of the car-following model.

Leutzbach has proved that the reaction time does not affect the formula when analyzing the macro traffic flow [20]. Therefore, it can be obtained as follows:

$$
\dot{u}=\lambda_{0} \frac{\dot{s}}{s^{1.5}}
$$

Density is the inverse of the distance between vehicles, then we can get:

$$
\int_{u}^{0} \dot{u}=-\int_{k}^{k_{j}} \lambda_{0} k^{1.5} \frac{\dot{k}}{k^{2}}
$$

Then:

$$
q=k u_{f}\left(1-\sqrt{\frac{k}{k_{j}}}\right)
$$

The basic graph of $q-k$ could be obtained as follows:

It can be seen from the Figure 2 at the beginning, the density is small and there is almost no mutual influence between vehicles. The flow increases almost linearly with the increase in density. After reaching the peak value, the flow starts to decrease with the increase in density. The state of traffic gradually turns into congestion and the mutual influence between vehicles increases. The peak $\left(k_{c h}, q_{h \max }\right)$ corresponds to the critical density and the maximum traffic flow of the road section, the line between any point on the curve and the origin corresponds to the average speed of vehicles at that point. The nonlinear car-following $(\alpha, \beta)$ model could qualitatively describe the traffic change characteristics of human-driven vehicles. Taking different values for simulation is better adapted to changes in different traffic environments. According to previous observations, the value of $\left(k_{c h}, q_{h \max }\right)$ is relatively stable. When the traffic flow approaches this value, it will cause the collapse of traffic, to some extent. When the flow reaches or approaches the critical point, the average of the traffic flow speed drops, traffic density increases and the density starts to develop in the direction of blocking density. When the flow reaches the capacity of the road, the traffic flow tends to be unstable and the available gap between vehicles becomes less. In this case, the disturbance caused by lane change or merging cannot be effectively weakened or eliminated. Mathematically, it corresponds to the upstream propagation of density disturbance which will cause traffic congestion or the occurrence of upstream queuing.

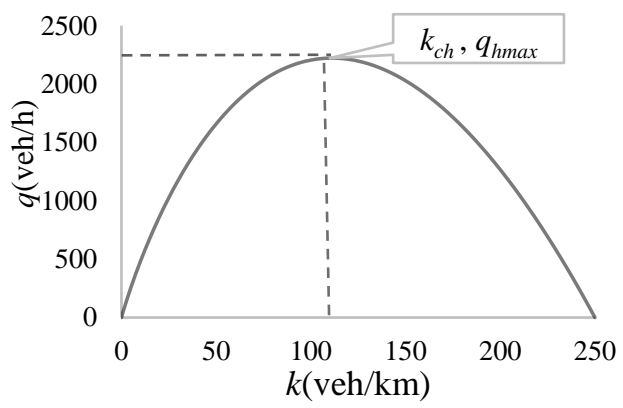

Figure 2. $q-k$ diagram with $p=0$. 


\subsection{Autonomous Vehicle Traffic Flow Model}

In a fully automatic driving road system, due to the high intelligence of the autonomous vehicle, it could always drive at the best speed in different situations of the whole driving process. Even by outside stimulation, it can automatically adjust and unify velocity in a very short time. Therefore, in a system of fully autonomous vehicles, the vehicle can always be coordinated to maintain a constant speed.

\subsubsection{Analysis of Following Characteristics of Autonomous Vehicle}

The model assumes that the vehicles travel at a constant speed without external stimulation. The length of all autonomous vehicles is $l$ and the reaction time $\Delta t$ is the same. Suppose that vehicle 1 is the front car, vehicle 2 follows vehicle 1 at speed $u$, vehicle 1 starts to decelerate with deceleration $a$ at $t=0$, and vehicle 2 follows vehicle 1 immediately after the reaction time. The safe distance $L$ is determined by anti-collision theory. The position of vehicle 1 is:

$$
s_{1}(t)=u t-\frac{1}{2} a t^{2} \quad\left(t \leq \frac{u}{a}\right)
$$

when $t \geq u / a$, vehicle 1 stops moving and will remain in the stop position $u^{2} / 2 a$. The following distance $L$ of vehicle 2 is:

$$
s_{2}(t)= \begin{cases}-L+u t & (t \leq \Delta t) \\ -L+u t-\frac{1}{2} a(t-\Delta t)^{2} & \left(\Delta t<t \leq \frac{u}{a}+\Delta t\right) \\ -L+\frac{u^{2}}{2 a} & \left(t>\frac{u}{a}+\Delta t\right)\end{cases}
$$

Then:

$$
s_{1}(t)-s_{2}(t)=\left\{\begin{array}{cc}
L-\frac{1}{2} a t^{2} & (t \leq \Delta t) \\
L+\frac{1}{2} a \Delta t^{2}-a t \Delta t & \left(\Delta t<t<\frac{u}{a}\right) \\
L+\frac{1}{2} a(t-\Delta t)^{2}+\frac{u^{2}}{2 a}-u t & \left(\frac{u}{a} \leq t<\frac{u}{a}+\Delta t\right) \\
L-u \Delta t & \left(t \geq \frac{u}{a}+\Delta t\right)
\end{array}\right.
$$

According to anti-collision theory, in a connected automatic driving system with a relative speed of 0 , if there is no reaction time, the vehicles could run synchronously and no collision will occur no matter how small the distance is. Therefore, the minimum distance $L$ should meet the following requirements: $L-u \Delta t \geq l$.

Equivalent deformation of the above equation could be obtained as follows:

$$
u \leq \frac{L-l}{\Delta t}
$$

Due to the smart characteristics of the autonomous vehicle, it could be set with optimized parameters. Therefore, when the road traffic density reaches a certain value and the vehicles start to influence each other, the following distance is set as: $L=u \Delta t+l$.

\subsubsection{Autonomous Vehicle Flow-Density Relationship}

When the following distance is not less than $L=u_{f} \Delta t+l$, vehicles could keep free-flow speed. Then:

$$
q=k u=k u_{f}
$$

That is, the traffic flow increases linearly with the increase of density, as shown in the OC phase in Figure 3. When the density reaches the critical density $k_{c w}$, then:

$$
k=k_{c w}=\frac{1}{u_{f} \Delta t+l}
$$




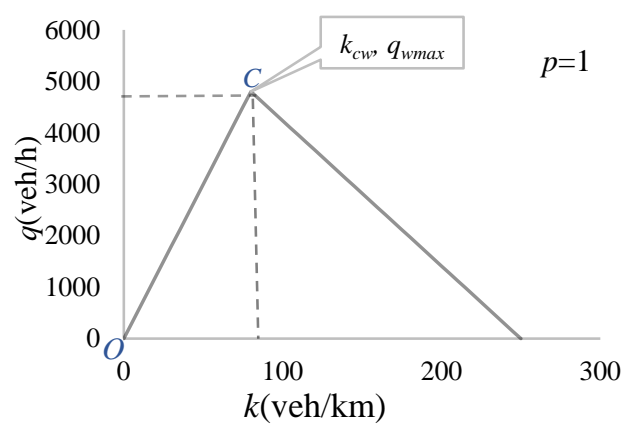

Figure 3. Flow-density diagram when $p=1$.

If the $\Delta t$ and speed do not change, the density and flow will not change. This will become the normal state of smart urban traffic in the future but it is not possible to be maintained in the actual traffic at present. When the system density is greater than the critical density, the autonomous vehicle starts to drive at speed $u$. Then the density is as follows:

$$
k=\frac{1}{u \Delta t+l}>k_{c w}=\frac{1}{u_{f} \Delta t+l}
$$

The flow rate changes linearly with the density, which is determined by the triangle basic diagram theory, namely:

$$
q=\min \left\{u k,\left(\frac{L-l}{\Delta t}\right) k\right\}
$$

When $k \leq k_{c w}$, the vehicle maintains free-flow speed; when the density is greater than $k>k_{c w}$, the speed begins to decrease with the increase of density, then:

$$
q= \begin{cases}k u_{f} & \left(k \leq k_{c w}\right) \\ \left(\frac{L-l}{\Delta t}\right) k & \left(k>k_{c w}\right)\end{cases}
$$

It could be concluded that the maximum density of the system with the speed of $u$ is: $1 /(u \Delta t+l)$. The maximum traffic flow of the system is:

$$
q^{\max }=u_{f} \frac{1}{u_{f} \Delta t+l}
$$

If there is a backward wave in the autonomous vehicle system, the speed of the backward wave is $w_{a}$ :

$$
w_{a}=-\frac{\frac{u_{f}}{u_{f} \Delta t+l}}{\frac{1}{u_{f} \Delta t+t}-\frac{1}{l}}=\frac{l}{\Delta t}
$$

The reaction time could not only affect the flow changes but also determine the maximum flow capacity of the system, which is an important reference index for the operating efficiency of the automatic driving system. If there is a backward wave, the magnitude of the backward wave is only related to the reaction time and the length of the vehicle.

\subsubsection{Flow-Density Relationship with Different Reaction Times}

From the above analysis, it could be concluded that the flow-density relationship with different reaction times is different during the driving process. In this paper, we assume that the length of the autonomous vehicle is $l=4 \mathrm{~m}$ and the free-flow speed is $u_{f}=60 \mathrm{~km} / \mathrm{h}$. Figure 4 shows the $q-k$ with different $\Delta t$ while other conditions remain unchanged when $p=1$. 


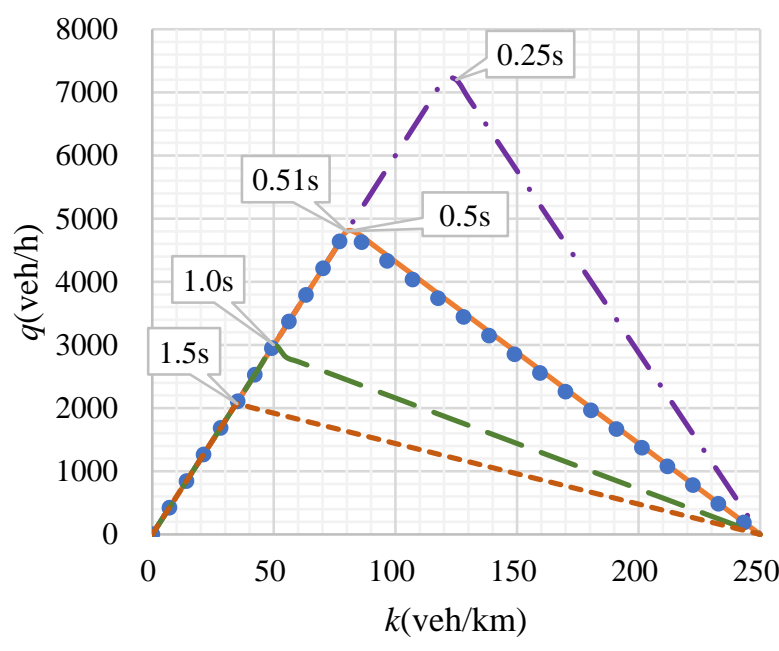

(a)

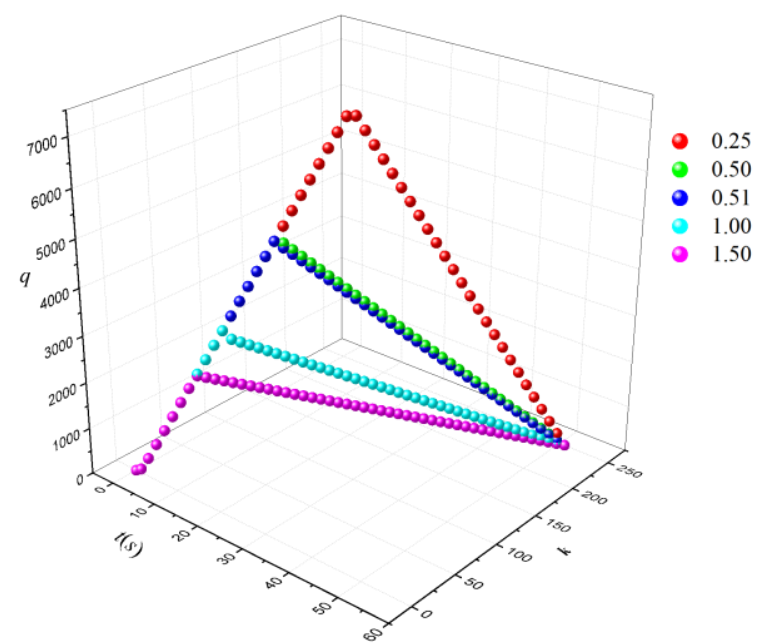

(b)

Figure 4. (a) Flow-density diagram for different reaction times, (b) time-flow-density diagram for different reaction times.

It could be seen from Figure $4 \mathrm{a}$ that, under the same road traffic conditions, the shorter the reaction time, the greater the critical density and the greater the traffic capacity. The reaction time and the maximum traffic volume have an opposite trend. When the reaction time is the same as the reaction time of a human-driven vehicle, the critical density and maximum traffic volume of the system are the same as human driving. In this paper, we take the reaction time of automatic driving as when the traffic flow at the critical density is about $4800 \mathrm{veh} / \mathrm{h}$. From Figure $4 \mathrm{~b}$, it can be concluded that the shorter the reaction time, the longer it takes to reach the critical density, the longer the system could maintain the free-flow speed, and the higher the road service level; the longer the reaction time, the sooner the critical density is reached, then vehicles need to slow down to maintain a safe following distance. When the reaction time of automatic driving is equal to human driving they reach critical density at the same time.

\subsection{Traffic Flow Analysis Model with Different Proportions}

In Sections 4.1 and 4.2, flow density models are established respectively. In this section, the theoretical derivation and analysis of the two models are carried out and the velocity variables are relaxed to describe the mixed traffic flow under the condition of different proportions of autonomous vehicles.

Assume that, under steady-state traffic conditions, human-driven vehicles are marked with " $\mathrm{H}$ " and the car-following distance is marked with $S_{h}$; autonomous vehicles are marked with " $\mathrm{W}$ ", and car-following distance is represented by $S_{w}$. In an operating system with moderate traffic conditions, the space headway under steady traffic conditions is shown in Figure 5:

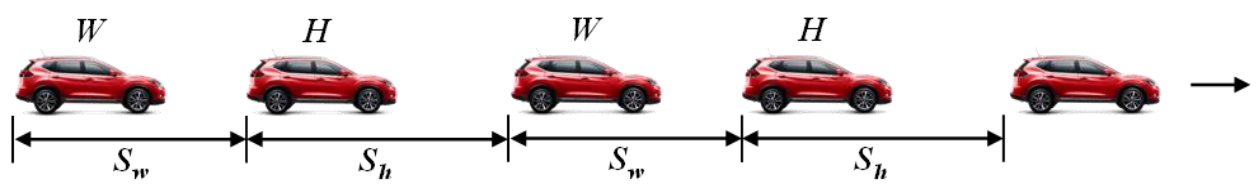

Figure 5. Schematic diagram of mixed traffic vehicle following.

Let $\bar{s}$ be the average inter-vehicle distance in the mixed traffic and $p \in[0,1]$ the proportion of autonomous vehicles in the system. Since there are only autonomous vehicles 
and human-driven vehicles in the research system, the proportion of human-driven vehicles is 1- $p$ and the average distance between vehicles in the system can be obtained as:

$$
\bar{s}=p s_{w}+(1-p) s_{h}
$$

From Section 4.2, when the following vehicle is an autonomous vehicle, the minimum safe distance between vehicles is:

$$
s_{w}=u \Delta t_{w}+l
$$

When the rear vehicle is a human-driven vehicle, the minimum safe distance between vehicles is:

$$
s_{h}=u \Delta t_{h}+l
$$

where $\Delta t_{h}$ is the average reaction time distribution of current human-driven vehicles.

In this article, due to the use of sensors and controllers of autonomous vehicles, as well as completely rational behavior, it is assumed that $\Delta t_{w}<\Delta t_{h}$. At a given speed, if the average length of the two vehicles is equal, then the total density of mixed traffic meets the following function:

$$
k_{\text {mix }}=\frac{1}{\bar{s}} \equiv f\left(p, s_{w}, s_{h}\right)
$$

In a mixed traffic system, when density is high, the autonomous vehicles could not run at a free-flow speed. So, in order to maintain the stable operation of the system, autonomous vehicles begin to match the speed of human-driven vehicles. That is, under steady traffic conditions, the speed of mixed traffic is determined by slower vehicles. Based on the above setting, the article adopts the reciprocal of the average inter-vehicle distance as the density of each type of vehicle. That is $k_{h}=1 / s_{h}, k_{a}=1 / s_{w}$. It can be seen that the density of each type of vehicle in mixed traffic is mutually determined.

We set $k_{h}$ and $k_{w}$ as the density of human and self-driving vehicles in mixed traffic respectively. When the system reaches a steady-state operation, the driving speed of mixed traffic is determined by the slow vehicles. As the autonomous vehicle is highly intelligent, it is a completely rational driver compared to a human driver and will not be easily disturbed by other external factors. An autonomous vehicle could always choose the optimal traffic speed according to the current road conditions. Due to the limitations of human driving, there will be some drivers with better driving skills who could maintain the same driving speed as automatic driving but most vehicle speeds are affected by other factors such as the reaction speed of human beings and the operation speed of vehicles. Therefore, in this paper, we limited $u_{h} \leq u_{w}$, the average speed of vehicles in mixed traffic under steady-state traffic is $u_{m i x}=u_{h}$.

Then:

$$
k_{\text {mix }}^{-1}=p k_{w}^{-1}+(1-p) k_{h}^{-1}
$$

So:

$$
q_{m i x}^{-1}=p q_{w}^{-1}+(1-p) q_{h}^{-1}
$$

Under steady-state traffic, the $q-k$ curve of mixed traffic is wrapped by $100 \%$ autonomous vehicles $q-k$ curve and $100 \%$ human driving vehicles $q-k$ curve. In a low-density state, autonomous vehicles and human-driven vehicles can maintain their respective speeds, there are $u_{h} \leq u_{m i x} \leq u_{w}$, then the relationship could still be maintained as shown in (29) by relaxing the velocity. No matter which speed is selected as the relaxed system speed, the relationship is satisfied. That is, the model is suitable for any traffic condition, but it could only show accurate numerical relationships under steady-state operating conditions. 


\subsection{Traffic Flow Model with Arbitrary Proportion of Autonomous Vehicles}

Section 4.3 is the relationship under steady-state traffic conditions. Although the relationship could still be satisfied after relaxing the vehicle speed conditions. However, it is not possible to calculate all traffic states relatively accurately. Based on this, combined with the multi-class hydrodynamics theory in Section 3.1, then according to the flow conservation conditions, a numerical model that satisfies any proportion of autonomous vehicles is established.

\subsubsection{Car-Following Analysis of Any Proportion of Autonomous Vehicles}

There are two types of vehicles in the research system of this paper: autonomous vehicles and human-driven vehicles, namely: $M=\{w, h\}$.

Where $w$ represents an autonomous vehicle and $h$ represents a human-driven vehicle. There are three main driving states of autonomous vehicles in a hybrid system:

(1) Keep driving at free-flow speed;

(2) Decelerate to match the driving state of human-driven vehicles;

(3) Keep pace with the speed of human-driven vehicles.

The above three states change gradually with the density of the traffic system from low to high. In different traffic systems, the density or time points of the three states change are different. Even if the data of countless samples are calibrated, it is impossible to accurately know what behavior human-driven vehicles will take in the next moment. However, with the current technology, it is easy to measure the relative position of human-driven vehicles with their own thorough sensors. Based on the principle of calculus, this paper establishes a car-following model of autonomous vehicles in a mixed traffic system. The model is suitable for any speed change of a human-driven vehicle.

The model is aimed at the car-following behavior that an autonomous vehicle could maintain when the speed of a human-driven vehicle changes at any time, the acceleration change of a human-driven vehicle could be set as a function $f_{a}(t)$. In order to ensure the vehicle will not retreat, when the speed is zero the acceleration is zero, and the acceleration changes for human-driven vehicles are:

$$
a_{h}(t)= \begin{cases}f_{a}(t) & \left(u_{h}>0\right) \\ 0 & \left(u_{h}=0\right)\end{cases}
$$

Then the velocity under any acceleration state is:

$$
u_{h}(t)=u_{h 0}+\int d u=u_{h 0}+\int_{t_{0}}^{t} a_{h}(t) d t
$$

The vehicle displacement is:

$$
s_{h}(t)=s_{h 0}+\int d s
$$

Then:

$$
s_{h}(t)=\int_{t_{0}}^{t} u_{h}(t) d t=\int_{t_{0}}^{t} \int_{t_{0}}^{t} a_{h}(t) d t d t
$$

According to:

$$
s^{\prime \prime}(t)=a(t)
$$

As the position of the next moment cannot be accurately predicted, only the position at time $t$ and before time $t$ could be known. Therefore, the known position of the humandriven vehicle is backward differed by using the backward Taylor expansion. 
The known backward Taylor expansion is:

$$
f^{\prime \prime}\left(x_{i}\right)=\frac{f\left(x_{i}\right)-2 f\left(x_{i-1}\right)+f\left(x_{i}-2\right)}{(\Delta x)^{2}}+O(\Delta x)
$$

Set the displacement at moment $t_{i}$ as $s\left(t_{i}\right)$, according to the Taylor expansion, then:

$$
a_{w}\left(t_{i}\right)=s_{h}^{\prime \prime}\left(t_{i}\right)=\frac{s_{h}\left(t_{i}\right)-2 s_{h}\left(t_{i-1}\right)+s_{h}\left(t_{i-2}\right)}{(\Delta t)^{2}}+O(\Delta t)
$$

When the autonomous vehicle in the system knows the position of the preceding vehicle at a certain moment, the speed or acceleration of the preceding vehicle could be calculated. In order to maintain the best following distance, the engineer could set the calculation program of the autonomous car-following model in the underlying program so that the autonomous vehicle and the human-driven vehicle can maintain synchronized speed changes.

Autonomous vehicles can set larger accelerations and decelerations through program calculations but, in actual travel, passengers' travel comfort needs to be considered or it could be completely replaced by full-speed sprints in a fully automatic driving system. Therefore, this paper refers to the light rail transit (LRT) and high-speed railway (HSR) [22,23] and the specified maximum speed change rate (acceleration and deceleration) of urban and expressway network as the standard for passenger comfort. Table 1 lists the maximum acceleration and deceleration change in the current study [23].

Table 1. Reference standards for acceleration and deceleration.

\begin{tabular}{ccc}
\hline & Acceleration & Deceleration \\
\hline Light rail transit & $1.34 \mathrm{~m} / \mathrm{s}^{2}$ & $-1.34 \mathrm{~m} / \mathrm{s}^{2}$ \\
High-speed rail & $0.58 \mathrm{~m} / \mathrm{s}^{2}$ & $-0.54 \mathrm{~m} / \mathrm{s}^{2}$ \\
Urban Dynamometer Driving Schedule & $1.5 \mathrm{~m} / \mathrm{s}^{2}$ & $-1.5 \mathrm{~m} / \mathrm{s}^{2}$ \\
Highway Economy Test Driving Schedule & $1.4 \mathrm{~m} / \mathrm{s}^{2}$ & $-1.5 \mathrm{~m} / \mathrm{s}^{2}$ \\
\hline
\end{tabular}

According to Table 1, set the maximum acceleration and deceleration value of autonomous vehicles in the urban road network as $\pm 1.5 \mathrm{~m} / \mathrm{s}^{2}$, The displacement of a humandriven vehicle from time $t_{0} \sim t$ meets:

$$
s_{h}(t)=s_{h 0}+\int d s=s_{h 0}+\int_{t_{0}}^{t} u_{h}(t) d t
$$

Assuming the relative distance between the autonomous vehicle and the humandriven vehicle is $d$, when the autonomous vehicle maintains free-flow speed, then the distance is:

$$
\left\{\begin{array}{c}
s_{w}(t) \leq s_{h}(t)+d \\
s_{w}(t)=u_{f} t
\end{array}\right.
$$

Suppose that the autonomous vehicle starts to be disturbed by the surrounding vehicles at $t_{\text {begin }}$, then slow down to adapt to the driving environment of the human-driven vehicle. The system starts to run at the same speed as the human-driven vehicle at $t$. Then, the position of the autonomous vehicle is as follows:

$s_{w}(t)=u_{f} t+\int_{u=t_{\text {begin }}}^{u=t} u_{w} d t=u_{f} t_{\text {begin }}+u_{f}\left(t-t_{\text {begin }}\right)-\frac{1}{2} a\left(t-t_{\text {begin }}\right)^{2}=u_{f} t-\frac{1}{2} a\left(t-t_{\text {begin }}\right)^{2}$

In order to prevent a collision, the vehicle shall meet the following requirements:

$$
\left\{\begin{array}{c}
d \leq s_{h}(t)-s_{w}(t) \\
u_{w}(t)=u_{h}(t)
\end{array}\right.
$$


Then get:

$$
\left\{\begin{array}{l}
u_{f} t-\frac{1}{2} a\left(t-t_{\text {begin }}\right)^{2} \leq s_{h}(t)+d \\
u_{f}-a\left(t-t_{\text {begin }}\right)=u_{h 0}+\int d u
\end{array}\right.
$$

Constraint condition:

$$
\left\{\begin{array}{c}
t \geq t_{\text {begin }} \\
0 \leq a \leq 1.5 \\
d \geq s_{w}(t)-s_{h}(t)
\end{array}\right.
$$

Considering the comfort of passengers, unless there are special circumstances, there should be no particularly sudden emergency change. Refer to Table 1 and select the optimal solution according to the actual traffic conditions. This model is suitable for any situation and could use different values in different traffic environments. In actual operation, the model can make the autonomous vehicle keep the same acceleration and deceleration behavior as the human-driven vehicle, when the system density reaches a certain value and the autonomous vehicle cannot change lanes or overtake in time.

\subsubsection{Traffic Flow Model with Different Proportion}

Let $k_{m}(x . t)$ be the density of $\mathrm{m}$-class vehicles at a space-time point $(x, t)$ according to the principle of hydrodynamics in Section 3, the total density is:

$$
k_{m i x}(x, t)=\sum_{m \in M} k_{m}(x, t)=k_{w}(x, t)+k_{h}(x, t)
$$

The flow $q_{w}$ of the autonomous vehicle at the time-space point $(x, t)$ is:

$$
q_{w}=q_{w}(x, t)=u\left(\frac{k_{w}}{k}\right) k_{w}(x, t)
$$

The flow $q_{h}$ of human-driven vehicles at the time-space point $(x, t)$ meets:

$$
q_{h}=q_{h}(x, t)=u\left(\frac{k_{h}}{k}\right) k_{h}(x, t)
$$

Functions $u\left(\frac{k_{w}}{k}\right)$ and $u\left(\frac{k_{h}}{k}\right)$ are the possible speeds when the proportions of autonomous vehicles and human-driven vehicles are $\frac{k_{a}}{k}$ and $\frac{k_{h}}{k}$, respectively.

According to the previous settings:

$$
p=\frac{k_{w}}{k}, 1-p=\frac{k_{h}}{k}
$$

The total traffic flow $q_{\text {mix }}(x, t)$ of the system at the time-space point $(x, t)$ is:

$$
q_{\text {mix }}(x, t)=\sum_{m \in M} q_{m}(x, t)=q_{w}(x, t)+q_{h}(x, t)=u_{w p}(x, t) k_{w p}(x, t)+u_{h p}(x, t) k_{h p}(x, t)
$$

Considering the actual traffic conditions, all the variables in this paper are bounded values not less than zero.

Based on this, an example of a $1 \mathrm{~km}$ section is analyzed in the fifth section, to verify the validity of the model.

\section{Experimental Analysis}

\subsection{Experimental Setting}

This article mainly studies the impact of autonomous vehicles on the current traffic flow, so even if some vehicles change lanes under balanced and steady traffic conditions it will not affect the overall distribution of traffic flow. For multiple lanes: if the density distribution between different lanes is not balanced, autonomous vehicles will give priority 
to driving in low-density lanes. If the vehicle could change lanes, the optimal system is that the autonomous vehicle becomes the leading vehicle through overtaking or lane change behavior. If the conditions for changing lanes and overtaking are not satisfied, the optimal system should be that the automatic driving vehicle maintains the same speed change as the human-driven vehicle through the speed change so as to reduce the car-following distance between vehicles and increase the capacity of the road, the redundant autonomous vehicle will spontaneously become a fleet driving, or autonomous vehicles and humandriven vehicles travel in separate lanes. What kind of behavior can make the system or user optimal cannot be directly judged. In view of this, the article sets the following scenarios for the case to analyze the impact of autonomous vehicles on road traffic changes:

I. The probability of the vehicle choosing different lanes to drive in is equal and the autonomous vehicle is a rational driver, changing lanes or overtaking does not bring better driving conditions. In this scenario, the law of traffic flow change between different lanes is the same, so analyzing one of the lanes is able to reflect the operating regulars;

II. Autonomous vehicles drive as the leader or become the leader in the low-density state by changing lanes or overtaking a few times;

III. Drive in separate lanes.

Based on this, this paper analyzes a two-lane road in the same direction that does not include diversion and merging. The length of the road section is $1 \mathrm{~km}$, time $T=50 \mathrm{~s}$, vehicle length $l=4 \mathrm{~m}$, the average reaction times are taken as $\Delta t_{h}=1.5 \mathrm{~s}, \Delta t_{w}=0.51 \mathrm{~s}$. The free flow speed is $60 \mathrm{~km} / \mathrm{h}$. The physical conditions of the lanes are the same and the probability of vehicles driving into different lanes is the same. For a certain lane, the blocking density is: $k_{j}=250 \mathrm{veh} / \mathrm{km}$; the critical density of autonomous vehicles is: $k_{c w}=80 \mathrm{veh} / \mathrm{km}$; the maximum flow of fully autonomous vehicles is: $q_{w}^{\max }=4800 \mathrm{veh} / \mathrm{h}$; the critical density of human-driven vehicles is: $k_{c h}=110 \mathrm{veh} / \mathrm{km}$; the human driving maximum flow is: $q_{h}^{\max }=2222 \mathrm{veh} / \mathrm{h}$.

\subsection{Numerical Results Analysis}

\subsubsection{Scenario Analysis}

For I scenario that may exist in Section 5.1 and the analysis in Section 4.4, the autonomous vehicle is mainly divided into three states. The following, Table 2 , shows some values of $p$ in the analysis of the calculation example.

Table 2. Partial values of the proportion $p$.

\begin{tabular}{lcccccccccccc}
\hline$w(\%)$ & 0 & 5 & 10 & 15 & 20 & 25 & $\begin{array}{l}\ldots \\
\ldots\end{array}$ & 80 & 85 & 90 & 95 & 100 \\
\hline$h(\%)$ & 100 & 95 & 90 & 85 & 80 & 75 & $\begin{array}{l}\ldots \\
\ldots\end{array}$ & 20 & 15 & 10 & 5 & 0 \\
\hline
\end{tabular}

It is generally regular when the autonomous vehicle initially drives on the road, or has a small proportion, or is under steady-state conditions where there is no overtaking or lane-changing behavior, and no dedicated lanes are set.

According to the theory of hydromechanics and the definition of density, no matter what the value of $p$ is, as long as they maintain the same physical characteristics as humandriven vehicles, the changes of density in mixed traffic over time remains consistent.

According to the model established in the fourth part, when $t_{\text {begin }}$ takes different values, $t$ and $a$ are shown in Table 3. 
Table 3. The values of $t$ and $a$ when $t$ begin take different values.

\begin{tabular}{cccccccc}
\hline $\boldsymbol{t}_{\boldsymbol{b}} \mathbf{( s )}$ & $\mathbf{0}$ & $\mathbf{1}$ & $\mathbf{2}$ & $\mathbf{3}$ & $\mathbf{3}$ & $\mathbf{4}$ & $\mathbf{5}$ \\
\hline$t$ & 12.10 & 10.98 & 10.03 & 9.21 & 9.00 & 8.51 & 7.90 \\
$\boldsymbol{a}$ & 0.68 & 0.78 & 0.93 & 1.15 & 1.18 & 1.52 & 2.28 \\
\hline
\end{tabular}

Taking into account safety and passenger comfort, referring to Table 1 for the current acceleration and deceleration standards in the traffic system, the calculation example in this paper is used $t_{\text {begin }}=3 \mathrm{~s}$, then takes $a=1.18 \mathrm{~m} / \mathrm{s}^{2}$ as acceleration to decelerate, until to the speed is synchronized with the human-driven vehicle at $t=9 \mathrm{~s}$, and then keep the same speed as the human-driven vehicle for the subsequent time. Figure 6 below shows the change of speed with density when autonomous vehicle follows human-driven vehicles in the traffic system set in this article.

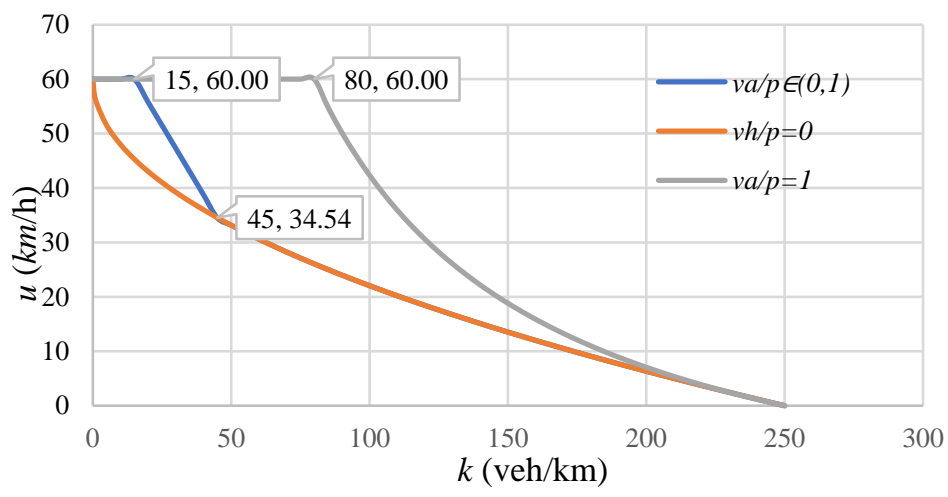

Figure 6. Speed fluctuation graph when $p$ takes different values.

If $p=1$, the autonomous vehicle could keep running at free-flow speeds until it reaches critical density; if $p \in(0,1)$, the speed of the autonomous vehicle is mainly divided into three stages:

- Free flow state;

- Decelerate to the same speed as the human-driven vehicle;

- Keep pace with the human-driven vehicle.

When the density is low, the speed of the autonomous vehicle is hardly disturbed by the distance between vehicles and the free-flow speed could be maintained. Humandriven vehicles are more susceptible to the influence of surrounding vehicles and the speed gradually decreases as the density increases. When the hybrid density reaches the level where the autonomous vehicle will be disturbed by the distance from the surrounding vehicles, the autonomous vehicle starts to decelerate with $a=1.18 \mathrm{~m} / \mathrm{s}^{2}$ until it is consistent with the speed of the human-driven vehicle. When $k_{\text {mix }}>45 \mathrm{veh} / \mathrm{km}$, the autonomous vehicle keeps driving at the same speed as humans. Figure 7 shows the speed-density change graph for $p=1$ and $p=0$.

It can be seen that, if $p=1$, the autonomous vehicle always maintains the free flow speed before reaching the critical density. When the density is greater than the critical density, the speed of the autonomous vehicle starts to decrease but it is still higher than the speed of all human-driven vehicles under the same density. If the system density gradually increases, traffic will gradually transition to a congested state, the distance between vehicles will become smaller, then the speed of autonomous vehicles will gradually tend to be the same as human-driven vehicles. The closer to the congestion density, the smaller the speed difference. When the system reaches the blocking density, both speeds are zero. 


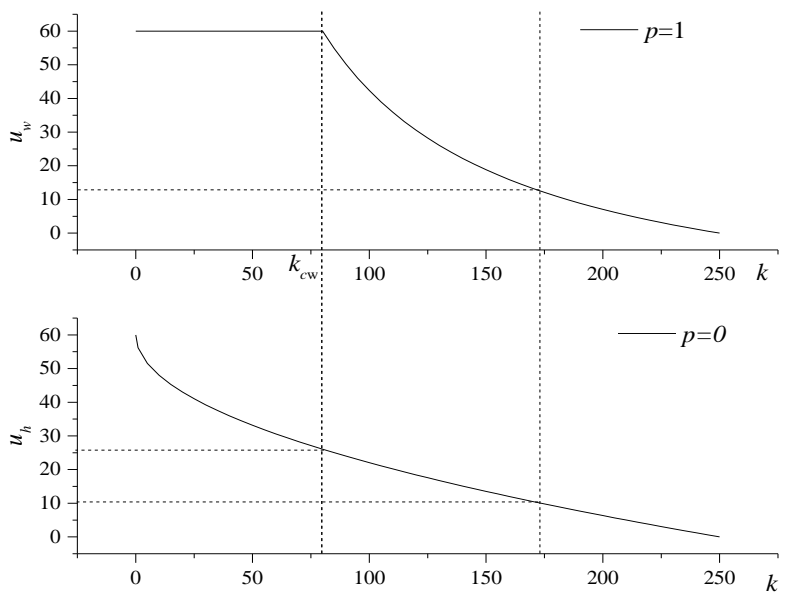

Figure 7. Speed-density diagram of $p=1$ and $p=0$.

Figure 8 shows the $q-k$ diagram when $p$ takes the value in Table 2 .

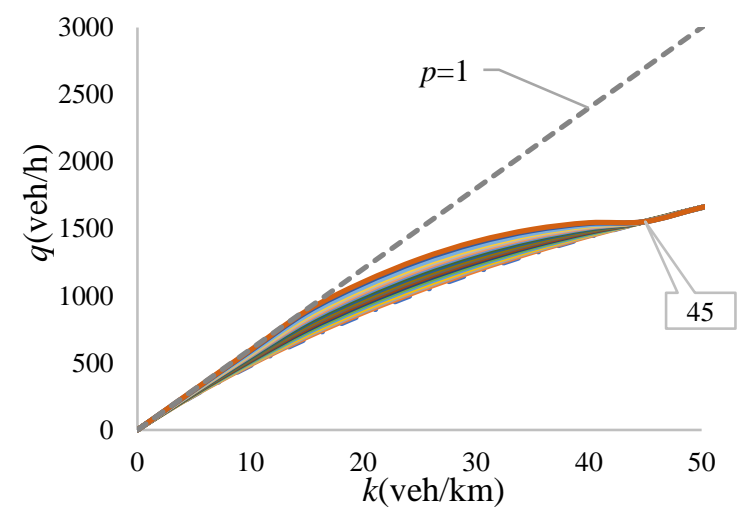

Figure 8. $q-k$ variation with different values of $p$.

From Figure 8, it could be concluded that when $p=100 \%$, the system has the highest travel efficiency. No matter what value $p$ takes, the mix $q-k$ curve is in the space between the autonomous traffic $q-k$ curve and human traffic $q-k$ curve. When $k_{\text {mix }} \leq 45 \mathrm{veh} / \mathrm{km}$, the system flow increases with the increase of $p$. When $k_{\text {mix }}>45 \mathrm{veh} / \mathrm{km}$, the system flow returns to the state of full human-driven vehicles. It can be known from Figure 8 that if the autonomous vehicle cannot be the leader of vehicles then, as long as $p \neq 1$, the maximum flow of the system cannot exceed the maximum flow of the human-driven vehicle. When system density is not large, the flow will increase with the increase of $p$. When the system starts to be congested until the congestion state, as long as there is a human-driven vehicle in the system the autonomous vehicle will match the human driving behavior. Therefore, the system will present a whole human driving state. If there is no better way to avoid congestion in time, even if the autonomous vehicle has a high proportion, the advantages of automatic driving cannot be effectively used.

From Figure 9 we could get that, if $p=1$, the system operating efficiency could be increased to about 2.2 times, which can improve the service level of the road. This will be an important step for smart cities in the future. Under the same traffic conditions, the fully autonomous vehicles can reach critical density earlier than all human-driven vehicles, that is to say, it has higher road utilization. The road conditions of the system cannot guarantee the autonomous vehicles will not be congested. Therefore, if the congestion in the road network could not be cleared in time, the automatic driving system will also present a state of blockage density. If the current system is all autonomous vehicles, it can bring huge potential benefits to society, such as reducing air pollution and improving work efficiency 
as analyzed in the introduction section. Figure 10 shows the change of flow with time when $p$ takes different values.

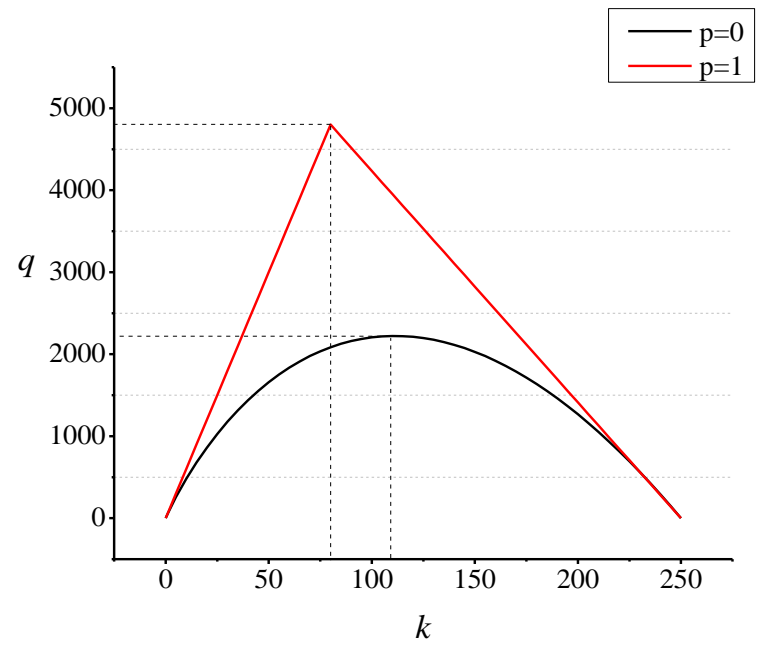

Figure 9. $q-k$ graphs with $p=0$ and $p=1$.

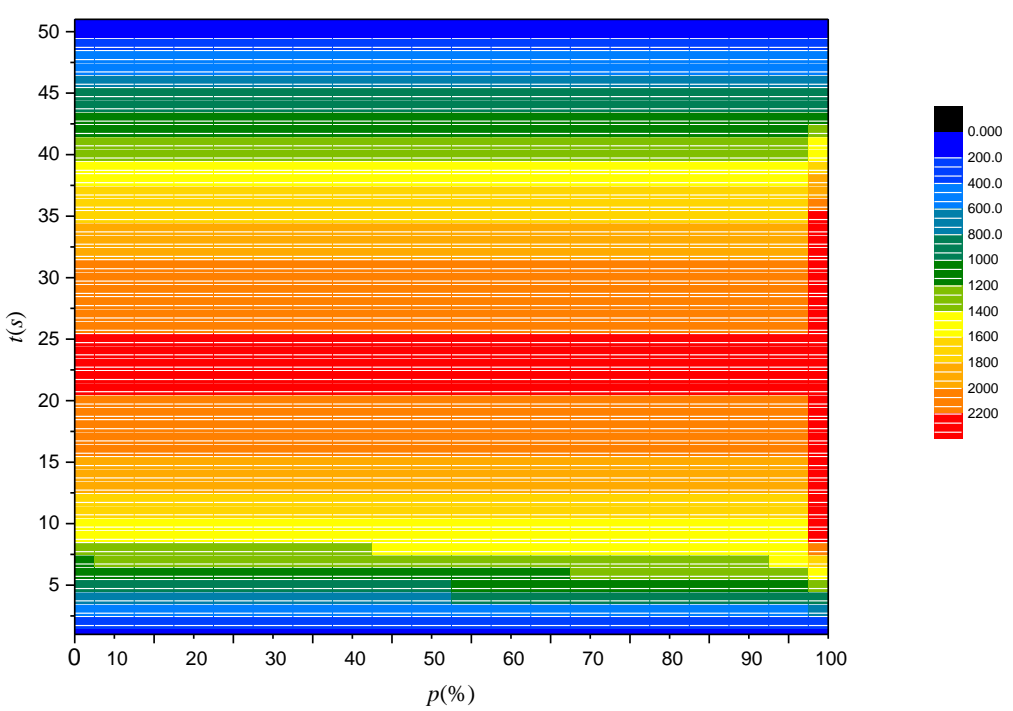

Figure 10. Time-flow variation with different $p$.

Table 4 shows some data of $q$ when $p$ takes different values in the actual analysis.

Table 4. Partial data of $q$ when $p$ takes different values.

\begin{tabular}{ccccccccc}
\hline $\boldsymbol{t}(\boldsymbol{s})$ & $\boldsymbol{p = \mathbf { 2 0 }}$ & $\boldsymbol{p = 4 0}$ & $\boldsymbol{p = 5 0}$ & $\boldsymbol{p = 6 0}$ & $\boldsymbol{p = 7 0}$ & $\boldsymbol{p = 8 0}$ & $\boldsymbol{p = 9 0}$ & $\boldsymbol{p = 1 0 0}$ \\
\hline 2.00 & 504.00 & 528.00 & 540.00 & 552.00 & 564.00 & 576.00 & 588.00 & 600.00 \\
3.00 & 723.64 & 767.73 & 789.77 & 811.82 & 833.86 & 855.91 & 877.95 & 900.00 \\
4.00 & 911.48 & 962.37 & 987.81 & 1013.26 & 1038.70 & 1064.15 & 1089.59 & 1200.00 \\
5.00 & 1078.05 & 1130.44 & 1156.63 & 1182.82 & 1209.02 & 1235.21 & 1261.41 & 1500.00 \\
$\vdots$ & $\vdots$ & $\vdots$ & $\vdots$ & $\vdots$ & $\vdots$ & $\vdots$ & $\vdots$ & $\vdots$ \\
48.00 & 290.94 & 290.94 & 290.94 & 290.94 & 290.94 & 290.94 & 290.94 & 282.35 \\
49.00 & 147.74 & 147.74 & 147.74 & 147.74 & 147.74 & 147.74 & 147.74 & 141.18 \\
50.00 & 0.00 & 0.00 & 0.00 & 0.00 & 0.00 & 0.00 & 0.00 & 0.00 \\
\hline
\end{tabular}

It could be known from Figure 10 that: $q_{h}^{\max }=2222 \mathrm{veh} / \mathrm{h}$ and $q_{w}^{\max }=4800 \mathrm{veh} / \mathrm{h}$. When the running time is more than $20 \mathrm{~s}$, regardless of the proportion of autonomous 
vehicles, the flow is above $2000 \mathrm{veh} / \mathrm{h}$. When the running time is greater than $25 \mathrm{~s}$, as long as $p \neq 1$, the traffic flow starts to drop below $2200 \mathrm{veh} / \mathrm{h}$. When $p=1$, the system flow can reach the maximum flow of all human driving in a short time, and maintain at least $q_{h}^{\max }=2222 \mathrm{veh} / \mathrm{h}$ to run for about $37 \mathrm{~s}$, after which the flow begins to decrease until it is blocked. In the early stage of system operation, at the same time period, the greater $p$, the greater $q$. When the system runs for more than $25 \mathrm{~s}$, the distance between vehicles begins to decrease, if the vehicle cannot leave the system in time, the system gradually transitions to a congested state. The larger $p$, the more traffic the road can carry before the autonomous vehicle matches the human-driven vehicle speed. The flow increases along the radius. As can be seen from Figure 11, under the same traffic conditions, the greater $p$, the greater disturbance that the autonomous vehicles will experience to match the speed of human-driven vehicles. In actual operation, taking into account the human-oriented and fully rational behavior of autonomous driving, the model established in this article can ensure the autonomous vehicle will not have a sharp change in speed during the process of matching human-driven vehicles, as shown in the radius direction there is no sharp fluctuation. It not only ensures travel comfort but also the stable operation of the system.

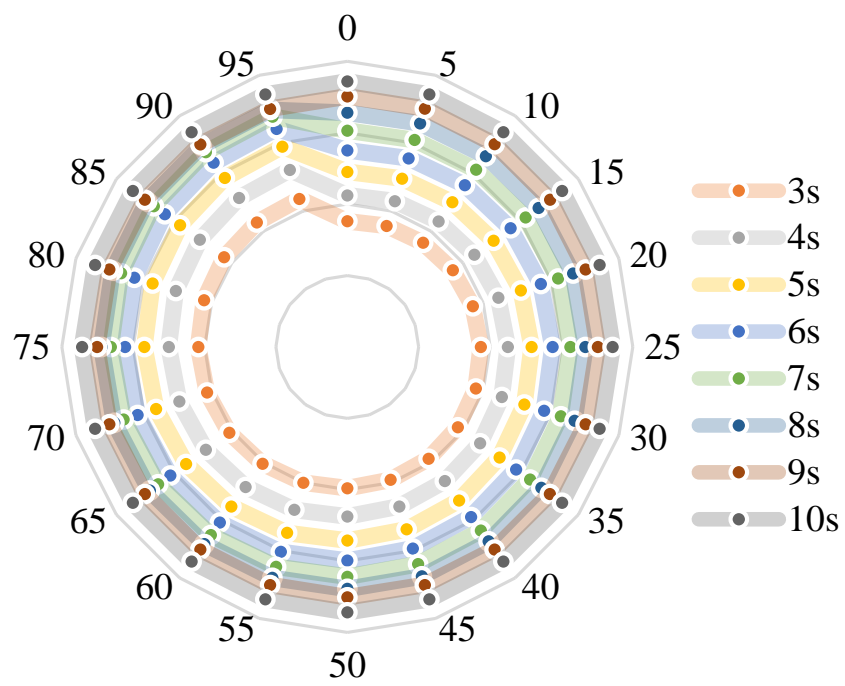

Figure 11. Flow fluctuation graph when $p$ takes different values.

As shown in Figure 12: when $p$ takes different values, the flow chart of the autonomous vehicle matching the speed of the human-driven vehicle. Table 5 shows some flow data of automatic driving to match human driving with different $p$ in the actual analysis.

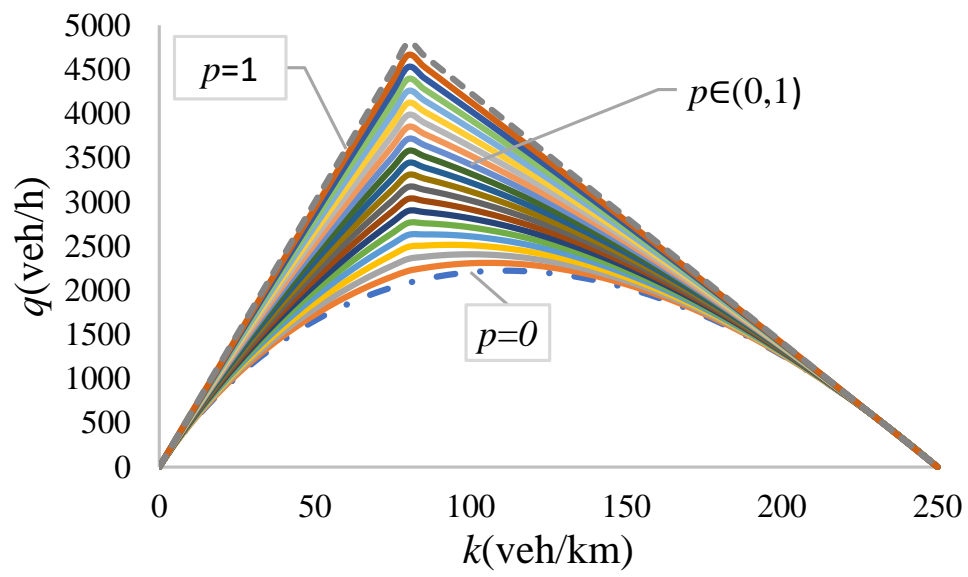

Figure 12. $q-k$ change with different $p$. 
Table 5. Matching data with different $p$ (partial data).

\begin{tabular}{cccccccccc}
\hline $\boldsymbol{p = 0}$ & $\boldsymbol{p = 1 0}$ & $\boldsymbol{p = 3 0}$ & $\boldsymbol{p = 4 0}$ & $\boldsymbol{p = 5 0}$ & $\boldsymbol{p = 6 0}$ & $\boldsymbol{p = 7 0}$ & $\boldsymbol{p = 8 0}$ & $\boldsymbol{p = 9 0}$ & $\boldsymbol{p = 9 5}$ \\
\hline 679.55 & 701.59 & 745.68 & 767.73 & 789.77 & 811.82 & 833.86 & 855.91 & 877.95 & 888.98 \\
679.546 & 701.591 & 745.682 & 767.728 & 789.773 & 811.818 & 833.864 & 855.909 & 877.955 & 888.977 \\
$\vdots$ & $\vdots$ & $\vdots$ & $\vdots$ & $\vdots$ & $\vdots$ & $\vdots$ & $\vdots$ & $\vdots$ & $\vdots$ \\
1658.36 & 1658.36 & 1658.36 & 1658.36 & 1658.36 & 1658.36 & 1658.36 & 1658.36 & 1658.36 & 1658.36 \\
\hline
\end{tabular}

In this section of research, the density is uniformly distributed. If special situations are not considered, the results of the above research will be presented in the universal state of the transportation system. Different parameter changes could be adopted in different traffic operating environments.

\subsubsection{Scenario Analysis}

In the same-direction multi-lane, if the density is low or the proportion of autonomous vehicles is relatively high, the autonomous vehicles could automatically evaluate the distance of overtaking and changing lanes and become the leading vehicle by overtaking or changing lanes few times. This scenario is mainly divided into two situations:

A. The autonomous vehicle just happens to lead the fleet after entering the system or leads the fleet after a few overtaking or lane changings;

B. In a low-density system, it becomes the leader through a few overtaking or lane changes.

For scenario A, Figure 12 shows that the traffic flow changes of the system when $p$ takes different values:

From Figure 12, we could still get that, if the proportion of autonomous vehicles is $100 \%$, the system has the highest travel efficiency. If $p \in(0,1)$, the mixed traffic flow q-k curve is still in the space between the autonomous traffic q-k curve and human traffic $\mathrm{q}-\mathrm{k}$ curve. The peak value of system traffic is positively correlated with the proportion of autonomous driving. Figure 12 shows that in a system where autonomous vehicles can lead a group, the maximum traffic flow increases with the increase of $p$. It also shows that, under low-density conditions, the system flow and peak value are positively correlated with the proportion of autonomous vehicles. The addition of autonomous vehicles can reduce travel time costs, improve system operating efficiency, reduce traffic pollution and improve the existing traffic environment.

Through the analysis of A and B in this section, it can be seen that if the autonomous vehicle is the leading vehicle in the system, regardless of the operating scenario, the flow will increase with the increase of $p$. If the density changes from low to high, the maximum flow will be also positively correlated with the value of $p$. No matter what the state, if $p=1$, the operation efficiency of the system is the highest. It could be seen that, when autonomous vehicles completely replace human-driven vehicles, the maximum traffic flow of fully autonomous vehicles is almost twice that of fully human-driven vehicles, which could greatly improve the urban transportation efficiency.

\subsubsection{Scenario Analysis}

When autonomous vehicles occupy half the proportion of the traffic system and vehicles can drive in separate lanes in road networks, the changes of road section traffic flow are shown in the following figure.

In the figure: - mix flow means one lane for autonomous vehicles, the other for human vehicles; -2lane-vh means the flow of human-driven vehicles in both lanes; -2lane-va means the flow of autonomous vehicles in both lanes. It can be seen from Figure 13a, in a system with divided lanes, the critical density of autonomous vehicles determines the critical density of the system, that is, the autonomous vehicles determine the maximum flow that the system may have. The traffic capacity of the fully automated driving system could reach 2.2 times that of all human-driven vehicles. If the autonomous vehicles and 
human vehicles drive in separate lanes, the flow can reach 1.56 times that of all humandriven vehicles. In a lane-separate system, when the proportion of autonomous vehicles is not less than the human-driven vehicles, the operating efficiency of autonomous vehicles determines the operating efficiency of the system.

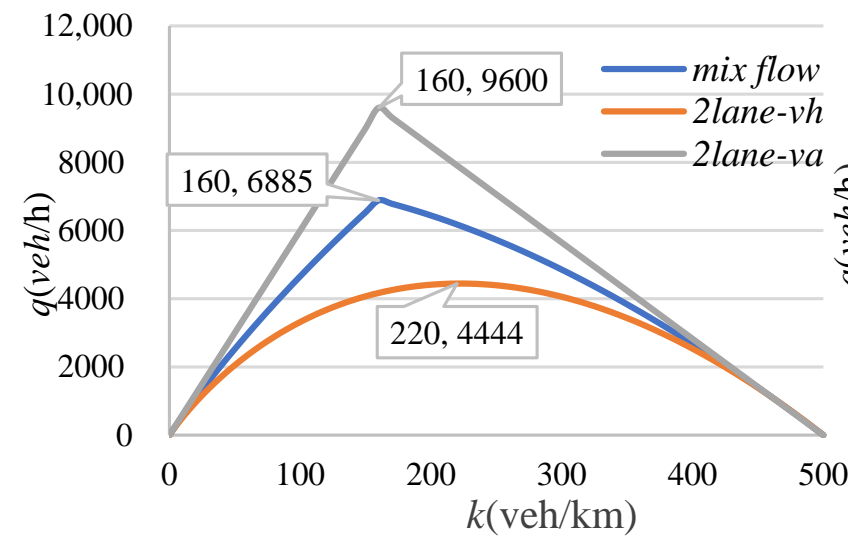

(a)

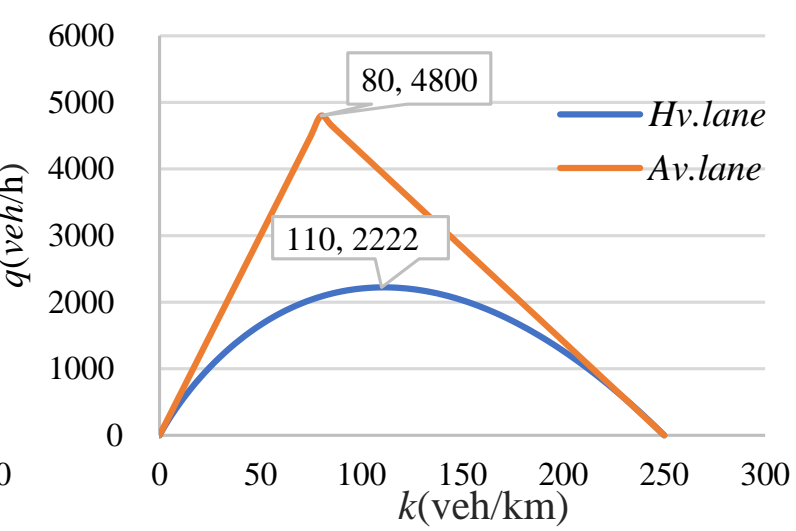

(b)

Figure 13. (a) $q-k$ variation chart in separate lanes, (b) $q$ in different lanes.

From Figure 13b, where human and automatic driving vehicles coexist, driving in separate lanes is the optimal strategy to improve the system operation efficiency.

Based on the above analysis, it could be concluded that the process of autonomous driving on the road gradually replacing human driving is also a process of gradually improving the efficiency of urban traffic.

\section{Conclusions}

With the background that autonomous vehicles will gradually replace human- driven vehicles in the next decades, we establish the shared road traffic flow models based on hydrodynamic theory, using calculus and difference methods. Then the participation of autonomous vehicles and how they influences the speed, density and flow of mixed traffic are analyzed. The main conclusions and contributions are as follows:

(1) If $p \in(0,1)$, the mixed traffic flow $q-k$ curve is in the region between the $q-k$ curve for fully human and autonomous traffic;

(2) Under different traffic scenarios, the addition of autonomous vehicles has different effects on the traffic system;

(3) The speed-following transformation model not only minimizes the safety distance but also saves road resources while optimizing the stability of transportation systems and could provide a necessary reference for the underlying programming design of autonomous vehicles;

(4) Establish a traffic flow model with arbitrary $p$ of autonomous vehicles;

(5) The reaction time of autonomous vehicles has a decisive influence on traffic flow;

(6) When autonomous vehicles occupy more than a half, split lane driving is the optimal strategy to improve the traffic capacity.

The research in this article could provide basic and necessary references for the distribution of traffic flow and road network design after autonomous vehicles driving on the road. At the same time, it could provide information on overall inventory, operating mode selection and traffic diversion and so on, which are of great significance for promoting autonomous vehicles driving on the road.

Author Contributions: Conceptualization, J.W.; Data curation, X.H.; Funding acquisition, G.B.; Methodology, H.L. All authors have read and agreed to the published version of the manuscript. 
Funding: This research received no external funding.

Institutional Review Board Statement: Not applicable.

Informed Consent Statement: Not applicable.

Data Availability Statement: The study did not report any data.

Conflicts of Interest: The authors declare no conflict of interest.

\section{References}

1. Levin, M.W.; Boyles, S.D. A multiclass cell transmission model for shared human and autonomous vehicle roads. Transp. Res. Part C Emerg. Technol. 2016, 62, 103-116. [CrossRef]

2. Lighthill, M.H.; Whithman, G.B. On kinematic waves II. A theory of traffic flow on long crowded roads. Proc. R. Soc. Lond. Ser. A. Math. Phys. Sci. 1955, 229, 317-334.

3. Richards, P.I. Shock Waves on the Highway. Oper. Res. 1956, 4, 42-51. [CrossRef]

4. Wong, G.C.K.; Wong, S.C. A Multi-Class Traffic Flow Model-An Extension of LWR. Transp. Res. Part A Policy Pract. 2002, 36, 827-841. [CrossRef]

5. Brackstone, M.; McDonald, M. Car-following: A historical review. Transp. Res. Part F Traffic Psychol. Behav. 1999, 2, 181-196. [CrossRef]

6. Gartner, N.H.; Messer, C.J.; Rathi, A.K. Revised Monograph on Traffic Flow Theory: A State-of-the-Art Report; National Transportation Library: Washington, DC, USA, 2001.

7. Newell, G. A simplified car-following theory: A lower order model. Transp. Res. Part. B Methodol. 2002, 36, 195-205. [CrossRef]

8. Kometani, E.; Sasaki, T. Dynamic Behaviour of Traffic with a Nonlinear Spacing-Speed Relationship. In Proceedings of the Symposium on Theory of Traffic Flow, Research Laboratories, General Motors, Warren, MI, USA, 7-8 December 1959; pp. 105-119.

9. Newell, G. A simplified theory of kinematic waves in highway traffic, part I: General theory. Transp. Res. Part B Methodol. 1993, 27, 281-287. [CrossRef]

10. Yperman, I.; Logghe, S.; Immers, B. The Link Transmission Model: An Efficient Implementation of the Kinematic Wave Theory in Traffic Networks. In Proceedings of the 10th EWGT Meeting, Poznan, Poland, 13-16 September 2005.

11. Le Vine, S.; Zolfaghari, A.; Polak, J. Autonomous cars: The tension between occupant experience and intersection capacity. Transp. Res. Part C Emerg. Technol. 2015, 52,1-14. [CrossRef]

12. Yu, Y.; Liu, S.; Jin, P.J.; Luo, X.; Wang, M. Multi-Player Dynamic Game-Based Automatic Lane-Changing Decision Model Un-der Mixed Autonomous Vehicle and Human-Driven Vehicle Environment. Transp. Res. Rec. 2020, 2674, 165-183. [CrossRef]

13. Wu, W.; Sun, R.; Ni, A.; Liang, Z.; Jia, H. Simulation and evaluation of speed and lane-changing advisory of CAVS at work zones in heterogeneous traffic flow. Int. J. Mod. Phys. B 2020, 34, 1-21. [CrossRef]

14. An, S.; Xu, L.; Qian, L.; Chen, G.; Luo, H.; Li, F. Car-following model for autonomous vehicles and mixed traffic flow analysis based on discrete following interval. Phys. A Stat. Mech. Appl. 2020, 560, 125246. [CrossRef]

15. Yang, H.; An, S. Robustness evaluation for multi-subnet composited complex network of urban public transport. Alex. Eng. J. 2021, 60, 2065-2074. [CrossRef]

16. Daganzo, C.F. The cell transmission model: A dynamic representation of highway traffic consistent with the hydrodynamic theory. Transp. Res. Part B Methodol. 1994, 28, 269-287. [CrossRef]

17. Daganzo, C.F. The cell transmission model, part II: Network traffic. Transp. Res. Part B Methodol. 1995, 29, 79-93. [CrossRef]

18. Daganzo, C.F. A finite difference approximation of the kinematic wave model of traffic flow. Transp. Res. Part B Methodol. 1995, 29, 261-276. [CrossRef]

19. Blumberg, M.; Bar-Gera, H. Consistent node arrival order in dynamic network loading models. Transp. Res. Part B Methodol. 2009, 43, 285-300. [CrossRef]

20. Leutzbach, W. Introduction to the Theory of Traffic Flow; Springer: Berlin/Heidelberg, Germany, 1972.

21. Bose, A.; Ioannou, P. Mixed manual/semi-automated traffic: A macroscopic analysis. Transp. Res. Part C Emerg. Technol. 2003, 11, 439-462. [CrossRef]

22. Testing and Measuring Emissions: Dynamometer Driving Cycles; US Environmental Protection Agency: Ann Arbor, MI, USA, 2014. Available online: http:/ / www.epa.gov/nvfel/testing/ (accessed on 4 June 2014).

23. Zhang, J.; Zhao, M.; Liu, H.; Xu, X. Networked characteristics of the urban rail transit networks. Phys. A Stat. Mech. Its Appl. 2013, 392, 1538-1546. [CrossRef] 